Sociologie et sociétés

\title{
Que se cache-t-il derrière les portraits statistiques nationaux ?
}

\author{
Le cas des Amérindiens au Québec
}

\section{What is Hidden behind the National Statistical Portraits? The Case of First Nations of Quebec}

\author{
Mélanie Deslauriers, Claire Durand et Gérard Duhaime
}

Volume 43, numéro 2, automne 2011

La statistique en action

Statistics in Action

URI : https://id.erudit.org/iderudit/1008242ar

DOI : https://doi.org/10.7202/1008242ar

Aller au sommaire du numéro

Éditeur(s)

Les Presses de l’Université de Montréal

ISSN

0038-030X (imprimé)

1492-1375 (numérique)

Découvrir la revue

Citer cet article

Deslauriers, M., Durand, C. \& Duhaime, G. (2011). Que se cache-t-il derrière les portraits statistiques nationaux ? Le cas des Amérindiens au Québec. Sociologie et sociétés, 43(2), 143-174. https://doi.org/10.7202/1008242ar
Résumé de l'article

Les recherches sur les conditions socioéconomiques des Autochtones sont dominées par la mise en parallèle de leur situation avec celle des Allochtones. L'autochtonité ou la résidence dans une collectivité des Premières Nations y sont implicitement mises de l'avant comme principaux facteurs explicatifs. La recherche présentée vise à circonscrire l'apport réel de ces deux facteurs en rendant manifeste la contribution d'autres facteurs pertinents. En utilisant les données censitaires de 2001, en réduisant la comparaison aux communautés voisines de celles des Premières Nations et en recourant aux analyses de régression, la recherche permet de montrer l'importance de l'environnement immédiat et de l'isolement géographique pour comprendre la situation des Premières Nations. Elle montre également l'importance de l'égalité des chances, en premier lieu l'accès à l'éducation, dans l'explication de l'accès à un emploi et à un revenu supérieur. Enfin, elle souligne l'ampleur du problème de surpeuplement des logements vécu dans certaines communautés. 


\section{Que se cache-t-il derrière les portraits statistiques nationaux?}

Le cas des Amérindiens au Québec

\section{MÉLANIE DESLAURIERS}

Département de sociologie

Université de Montréal

C. P. 6128 , succursale Centre-ville

Montréal (Québec) $\mathrm{H}_{3} \mathrm{C}_{3} / 7$

Courriel:melanie.deslauriers.2@umontreal.ca

\section{CLAIRE DURAND}

Département de sociologie

Université de Montréal

C. P. 6128 , succursale Centre-ville

Montréal (Québec) $\mathrm{H}_{3} \mathrm{C}_{3} / 7$

Courriel: claire.durand@umontreal.ca

\section{GÉRARD DUHAIME}

Département de sociologie

Université Laval

1030, avenue des Sciences-Humaines

Québec (Québec) GıV oA6

Courriel: gerard.duhaime@soc.ulaval.ca

\section{INTRODUCTION ${ }^{1}$}

La recherche quantitative sur les conditions de vie des Autochtones s'est accrue à la suite de la Commission royale sur les peuples autochtones de 1992. Cela a permis de rendre plus manifestes dans l'espace public canadien les inégalités vécues par les

* Nous tenons à remercier le Conseil de recherches en sciences humaines du Canada (CRSH) ainsi que le Centre interuniversitaire québécois de statistiques sociales (CIQSS) pour l'aide financière accordée à cette recherche. Nous tenons également à remercier les évaluateurs anonymes pour leurs commentaires avisés et détaillés qui ont grandement aidé à améliorer le manuscrit.

1. Bien que la recherche et les analyses soient fondées sur des données de Statistique Canada, les opinions exprimées ne représentent pas celles de Statistique Canada. 
Autochtones $^{2}$. Toutefois, la recherche quantitative s'est concentrée sur la mise en parallèle des conditions socioéconomiques des Autochtones et des Allochtones sans égard aux facteurs internes qui fragmentent ces deux catégories sociales. Ainsi, l'autochtonité apparaît comme le principal déterminant des inégalités puisqu'il est le principal, voire le seul facteur énoncé dans ces recherches, habituellement strictement descriptives.

Édouard (2008) souligne que ces analyses, bien qu'utiles, nous emprisonnent dans des pièges éthiques et épistémologiques. En plus de mettre de l'avant une conception de la société canadienne caractérisée principalement par un clivage fondamental opposant les Autochtones et le reste de la population, elle pose la situation des Allochtones en modèle à suivre pour les Autochtones et engendre une conception linéaire du développement où ces derniers seraient à la traîne des autres Canadiens. La mise en comparaison Autochone-Allochtone a pour conséquence de réifier ces groupes en donnant l'impression qu'ils sont homogènes et dénués de tout clivage interne. Elle renforce l'image que ces catégories vont de soi plutôt que de mettre de l'avant qu'elles sont la résultante de processus historiques.

La perspective du nominalisme dynamique (Hacking, 2006) permet de contourner l'idée de la «réalité», au sens métrologique, de ces deux groupes tout en justifiant qu'on s'attarde à ceux-ci. Les catégories utilisées par le dispositif statistique d'État, mais aussi par l'appareil législatif, pour procéder à la division du monde social finissent par "façonner les gens» (Hacking, 2006), c'est-à-dire modeler la façon dont ces derniers se pensent et organisent leur expérience de la réalité. C’est donc en partie dans l'exercice du recensement et de ce qui en découle que cette labellisation acquiert une partie de sa légitimité et de son caractère «véridique». Dans la mesure où Autochtone et non-Autochtone sont des catégories fréquemment utilisées dans le sens commun et qu'elles deviennent le point focal de la plupart des recherches quantitatives traitant des questions amérindiennes (voir entre autres Gionet, 2008; Statistique Canada, 2008d; George et Khun, 1994; AINC, 1997; Maxim, White et Beavon, 2001), il devient justifié de s'interroger sur l'apport explicatif unique du fait d'«être autochtone» dans les disparités socioéconomiques.

Cet article se concentre sur un seul des trois groupes autochtones reconnus officiellement, soit les Amérindiens. Il vise à examiner la contribution des facteurs de contexte et des facteurs individuels tels que l'âge et le genre dans la compréhension des dynamiques d'inégalités sociales attribuées à l'autochtonité ou à la résidence dans une communauté des Premières Nations. Ce faisant, la recherche va au-delà des distinctions habituelles Amérindien - non-Amérindien en examinant d'une part où se concentrent les inégalités entre les deux «groupes» et d'autre part, jusqu'à quel point celles-ci s'expliquent par des contextes de vie communs ou différenciés. La première

2. Le terme Autochtone fait référence aux trois groupes reconnus officiellement dans la loi constitutionnelle de 1982, soit les Amérindiens, les Inuits et les Métis. 
question à résoudre est toutefois celle de la définition statistique de qui est amérindien et qui ne l'est pas.

\section{QUI EST AMÉRINDIEN ? DIFFÉRENTES CONCEPTIONS STATISTIQUES}

A priori, on pourrait penser que les critères départageant les individus entre Amérindiens et «autres Canadiens» vont de soi et sont stables à travers le temps tant cette «boîte noire» qu'est le groupe «Amérindien» est présentée comme une entité homogène dans la recherche sur les conditions autochtones. Les questions relatives à l'identification de qui est ou n'est pas considéré amérindien sont généralement passées sous silence. Toutefois, la composition de cette catégorie diffère, d'une part, selon les auteurs et, d'autre part, avec le temps. Ceci est en partie dû au fait que le nombre et le libellé des questions relatives à l'autochtonité fluctuent d'une enquête censitaire à l'autre.

La population «amérindienne» du recensement de 1986 est constituée des individus ayant déclaré une origine amérindienne à la question sur l'ascendance/l'origine ethnique, que ce soit comme réponse unique ou associée à d'autres origines. En 1991, s'ajoutent à ces individus ceux ayant déclaré un statut d'Indien inscrit, qu'ils aient ou non déclaré des origines amérindiennes à la question sur l'ascendance. Dès l'enquête de 1996, les questions relatives à l'identification comme «Indien de l'Amérique du Nord», la déclaration d'un statut d'Indien inscrit/d'Indien des traités ou l'appartenance à une Première Nation sont introduites dans la construction de la population. Finalement, depuis 2001, les mêmes quatre questions sont utilisées, mais le libellé de la question sur l'origine a été légèrement modifié. Par ailleurs, pour un même recensement, la population amérindienne étudiée par les auteurs n'est pas toujours la même. Gerber (1990) ainsi que George et Kuhn (1994) utilisent la question d'origine 3 , Wilkes (2010), la question d'identification, Bernier (1999), conjointement ces dernières questions ${ }^{4}$, alors qu'Affaires Indiennes et du Nord Canada (AINC) (1997) utilise le statut juridique et le lieu de résidence. Enfin, certains auteurs ne prennent en compte que les réponses simples, à l'exclusion des réponses multiples.

Le choix d'une mesure n'est pas une considération méthodologique de second ordre puisqu'elle renseigne sur la conception même de l'autochtonité. Guimond (2003) a montré l'importance de se questionner sur les critères de construction de «la population autochtone» et ce, d'autant plus qu'il n'existe pas de consensus sur ce qui constitue l'autochtonité dans le contexte canadien. Le croisement des réponses aux questions correspondant aux principaux concepts utilisés — origine, identité et statut juridique - montre qu'il n'existe pas de structure hiérarchique de ces dimensions.

3. Gerber (1990) restreint ce concept aux individus ayant déclaré des origines maternelles et paternelles amérindiennes, inuites ou métisses.

4. Une comparaison est effectuée, entre autres, entre la population d'origine autochtone et d'identité. La population d'identité correspond aux individus ayant déclaré une ou plusieurs origines et une ou plusieurs identités autochtones et non pas uniquement une identité, ce qui la distingue de l'étude de Wilkes (2010). 
Plutôt que de se solder par la formation de groupes emboîtés les uns dans les autres, comme des poupées gigognes, la mise en commun des réponses aux trois questions aboutit à un enchevêtrement partiel des dimensions. Certains répondants déclarent une ascendance autochtone sans s'identifier à un groupe autochtone, l'inverse étant aussi possible. De plus, certains Indiens inscrits ne déclarent pas d'origine autochtone (par exemple certaines femmes allochtones mariées à un «Indien inscrit» avant 1985), mais s'identifient tout de même comme Amérindiens.

\section{COLLECTIVITÉS DES PREMIÈrES NATIONS, PARTICULARITÉS JURIDIQUES ET ORGANISATION SCOLAIRE}

Au Canada, certains territoires, nommés réserves ${ }^{5}$, ont été gardés à l'usage exclusif des Amérindiens. Les réserves sont très hétérogènes tant dans leur superficie que dans la diversité des ressources naturelles qu'elles contiennent. Mis à part quelques exceptions, les communautés ne possèdent qu'un droit collectif d'usage des terres, ces dernières étant la propriété du gouvernement, et ce droit ne leur est consenti que tant qu'elles occupent le territoire (Dupuis, 1998). Par extension, les résidants des réserves ne sont propriétaires ni de leur habitation, ni de la terre sur laquelle celle-ci est construite (Dupuis, 1998). Le financement des réserves se fait en partie via les transferts gouvernementaux. Ceux-ci dépendent, entre autres, de l'emplacement géographique de la communauté et du nombre de résidants avec statut que celle-ci comporte. Pour pouvoir utiliser les fonds qui leur sont alloués, les communautés doivent faire approuver les retraits par le Ministère (Dupuis, 1998).

Les réserves sont donc, en pratique, sous tutelle. La Loi sur les Indiens donne un pouvoir considérable au gouvernement fédéral dans l'administration de la vie collective des collectivités des Premières Nations, pouvoir qui n'a pas d'égal dans les autres municipalités canadiennes. Ceci limite le champ d'action des collectivités sur le plan administratif et peut s'avérer contraignant pour la réalisation des travaux publics ou le développement de la communauté.

En ce qui concerne l'organisation scolaire, l'administration et la construction des écoles dans les collectivités des Premières Nations étaient, jusqu'en 1978, sous la responsabilité du ministère des Affaires indiennes et du Nord Canada. De façon générale, les enfants fréquentaient une école administrée par le gouvernement fédéral pour le primaire puis étaient inscrits dans des écoles secondaires ou postsecondaires du réseau public québécois (ministère de l'Éducation, du Loisir et du Sport, 2004). À partir de 1978, une prise en charge progressive de l'éducation par les communautés des Premières Nations s'est amorcée au Québec, alors que les conseils de bande ${ }^{6}$ ont assumé la gestion des écoles sur leur territoire et qu'il leur est devenu possible d'adap-

5. Le terme de réserve n'est pas nécessairement apprécié, entre autres par les Premières Nations parce qu'il comporte une image négative. Toutefois, l'utilisation du terme dans ce texte permet de rappeler la situation juridique différenciée à laquelle font face les collectivités des Premières Nations.

6. Les conseils de bande sont les organismes élus qui sont responsables de la gestion des réserves. 
ter les programmes d'enseignement, notamment en ce qui a trait à la langue d'enseignement au début du primaire. Un déplacement complet des élèves fréquentant les écoles fédérales vers les écoles de bande ainsi qu'une diminution progressive du nombre d'élèves qui fréquentent le réseau scolaire public québécois ou une école privée s'est opéré depuis les dernières années. Les services d'éducation primaire et secondaire — voire dans certains cas la formation aux adultes — sont aujourd'hui gérés par les communautés dans la majorité des cas et financés par le gouvernement fédéral en vertu de la Loi sur les Indiens, sauf pour les Cris et les Naskapis qui sont signataires de conventions spécifiques.

\section{LES GRANDES TENDANCES DE LA RECHERCHE QUANTITATIVE SUR LES CONDITIONS SOCIOÉCONOMIQUES DES AUTOCHTONES}

Trois niveaux de comparaison peuvent être dégagés de la recherche quantitative portant sur les conditions socioéconomiques des Autochtones, soit les analyses pancanadiennes, provinciales et intra-provinciales ou régionales. Dans les analyses pancanadiennes, la recherche porte principalement sur les disparités socioéconomiques globales entre Autochtones et Allochtones. L'espace d'équivalence est le territoire canadien dans son ensemble et à l'intérieur de cet espace, les expériences des Autochtones et des «autres Canadiens» sont mises en parallèle. On y retrouve, entre autres, les recherches de Gerber (1990), de George et Kuhn (1994), de Maxim et White (2001), de Bernier (1997), de De Silva (1999), d'AINC (1997), de Wotherspoon (2003), de McHardy et O'Sullivan (2004), de O'Sullivan et McHardy (2004), de White et Maxim (2007) et de Wilkes (2010), toutes réalisées à partir de données de recensement. Ces analyses montrent la présence de disparités socioéconomiques selon l'appartenance ethnique - Autochtones vs Allochtones —, mais aussi selon le genre (Gerber, 1990), l’âge (De Silva, 1999), la scolarité (Wilkes, 2010; De Silva, 1999), le statut politico-légal — «Indiens inscrits» vs les non-inscrits — (Maxim et White, 2001) ainsi que le type de communauté habitée - réserve ou non - (O'Sullivan et McHardy, 2004) ou son degré d'isolement (White et Maxim, 2007; McHardy et O’Sullivan, 2004; AINC, 1997).

Certains auteurs (Maxim et White, 2001; Bernier, 1997; Gerber, 1990) ont fragmenté la catégorie "Autochtone» afin de tenir compte des trois différents "peuples» autochtones reconnus dans la Loi constitutionnelle de 1982. Cela a permis de faire apparaître les disparités salariales et de revenu existant entre les Amérindiens, les Métis et les Inuits, mais également au sein de chacune de ces catégories (Maxim et White, 2001; Bernier, 1997; Gerber, 1990). De plus, Maxim et White (2001) notent une tendance générale à l'accroissement des disparités de revenus, plus marquée chez les Amérindiens, les Métis et, surtout, les Inuits que chez les Allochtones.

D'autres recherches (White et Maxim, 2007; McHardy et O’Sullivan, 2004; O’Sullivan et McHardy, 2004; AINC 1997) se sont penchées sur les disparités dans les conditions socioéconomiques entre les diverses collectivités des Premières Nations, mais également entre ces dernières et les autres collectivités canadiennes. Elles ont 
montré l'importance de l'isolement (habituellement mesuré par la zone géographique d'appartenance - urbaine, rurale, isolée ou éloignée - établie par l'AINC selon les critères de proximité et d'accessibilité routière aux centres de services) comme facteur déterminant des conditions socioéconomiques, son effet étant toutefois plus marqué pour les collectivités des Premières Nations que pour les autres collectivités (White et Maxim, 2007).

White et Maxim (2007), McHardy et O’Sullivan (2004) et O'Sullivan et McHardy (2004) ont analysé la relation entre le degré d'isolement et l'indice de bien-être des collectivités $\left(\mathrm{IBC}^{7}\right)$, pris globalement ou par sous-dimension. Ces recherches ont montré que la moyenne de l'IBC des collectivités en zone urbaine était la plus élevée et celle des zones éloignées la plus basse. Elles ont également mis au jour l'étendue de la variation dans le bien-être des communautés, qu'elles soient ou non des collectivités des Premières Nations (McHardy et O'Sullivan, 2004).

White et Maxim (2007), en jumelant 495 communautés des Premières Nations avec des collectivités jugées similaires par leur taille et leur emplacement, ont permis de nuancer les résultats obtenus sur les valeurs totales d'IBC. Ils ont montré que la valeur moyenne de l'IBC scolarité des collectivités des Premières Nations est largement supérieure en zones urbaine et rurale que dans les autres zones alors que celle de l'IBC activité est sensiblement la même pour les zones urbaine et isolée. Toutefois, l'écart dans les valeurs d'IBC activité et revenu entre les collectivités des Premières Nations et les collectivités comparables s'est révélé plus faible en zone isolée qu'en zone urbaine. Quant à la qualité et à la quantité des logements, elles semblent se dégrader avec l'isolement, et ce, pour les deux types de communauté. De façon générale, les écarts entre les réserves et les collectivités comparables apparaissent moins grands à mesure que l'on se rapproche des centres de services (White et Maxim, 2007).

Cependant, les études longitudinales de l'AINC (1997), réalisées à partir des recensements de 1971 à 1991, et de McHardy et O'Sullivan (2004), effectuées à partir des recensements de 1991 à 2001, concluent que l'écart dans les conditions socioéconomiques selon le type de communauté persiste mais tend à s'amenuiser avec le temps. De façon générale, les conditions de vie se sont améliorées dans l'ensemble des collectivités canadiennes entre 1991 et 2001, mais cet accroissement a été plus marqué dans les collectivités amérindiennes (McHardy et O'Sullivan, 2004).

Le deuxième niveau de comparaison s'attache aux différences dans les conditions de vie qu'offrent les diverses provinces canadiennes. McHardy et O'Sullivan (2004) et O’Sullivan et McHardy (2004) montrent que les écarts dans l'IBC total entre les collectivités des Premières Nations et les autres collectivités varient selon les provinces. Ils sont plus marqués dans les provinces de l'Ouest, et plus particulièrement en Alberta, que dans les autres régions alors qu'ils sont à leur plus bas niveau dans les

7. L'IBC est un indice synthétique inspiré de l'indice de développement humain (IDH) créé par le Programme des Nations Unies pour le développement (PNUD) pour évaluer le niveau de développement des pays selon trois critères: l'espérance de vie, le niveau d'éducation et le niveau de vie. L'IBC comprend, outre un indice global, quatre sous-indices portant sur la scolarité, l'activité, le revenu et le logement. 
régions de l'Atlantique et du Nord. Par ailleurs, O'Sullivan et McHardy (2004) font ressortir que, de 1991 à 2001, la plus petite réduction de cet écart est observée dans le Nord alors que les plus importantes réductions se sont produites en Saskatchewan et au Québec.

L'étude de Wilkes (2010), basée sur les données censitaires de 2001, permet de rendre compte à la fois de la persistance de disparités socioéconomiques entre Autochtones et Allochtones dans l'ensemble du Canada et de leur ampleur variable selon les provinces. À titre d'exemple, le pourcentage d'Autochtones n'ayant pas terminé leurs études secondaires était particulièrement élevé dans quatre provinces et territoires - le Québec, le Manitoba, la Saskatchewan et les Territoires du NordOuest. Au Québec, ce pourcentage était de 51,6\%, comparativement à 31,5\% chez les Allochtones (Wilkes, 2010). Par ailleurs, pour ce qui est du revenu, les écarts les plus importants entre Autochtones et Allochtones se situent au Nunavut alors qu'ils sont à leur plus bas niveau à Terre-Neuve et Labrador (Wilkes, 2010). Enfin, les écarts dans la participation au marché du travail sont plus marqués dans les Prairies et dans le Nord que dans les autres provinces. Au Québec, cet écart était de 6,5\%, le taux de participation des Autochtones se situant à 57,7\% et celui des Allochtones à 64,2\%.

Le troisième niveau de comparaison est caractérisé par la mise de l'avant de conditions socioéconomiques différenciées au sein d'une province ou d'une sous-région d'une province (Édouard, 2008; Duhaime et Godmaire, 2002; Simard, 2003 ; Kulshreshtha et Dore, 2003). Pour ce qui est des communautés des Premières Nations du Québec, Duhaime et Godmaire (2002) ont proposé une typologie permettant de classer les communautés selon, d'une part, le type d'activité économique dominante (grande échelle vs petite échelle) et, d'autre part, l'appartenance ethnique principale des membres (Allochtone vs Autochtone). En comparant les quatre types d'aires obtenues par le croisement du type d'échelle et du type de communauté, ils montrent que les revenus par ménage sont plus élevés dans les aires autochtones d'économie à grande échelle (régions minières ou forestières, zones de construction des barrages hydro-électriques) que dans les aires allochtones d'économie à petite échelle tels les villages de pêcheurs. Les aires autochtones d'économie à grande échelle comprennent des communautés sédentarisées depuis peu qui ont conclu des ententes pour pouvoir exploiter les ressources naturelles situées sur leur territoire. Elles sont caractérisées entre autres par un niveau de dépense publique élevé à la suite des conventions signées qui ont facilité la construction d'infrastructures scolaires et de santé. Les aires allochtones d'économie à petite échelle présentent un niveau de scolarisation peu élevé et un revenu par ménage qui se situe près de la moyenne du Québec isolé; elles sont tributaires principalement de l'apport de transferts gouvernementaux.

En résumé, les recherches montrent que les collectivités des Premières Nations offrent généralement des conditions de bien-être inférieures à celles offertes en moyenne par les autres collectivités, que la comparaison se fasse sur le plan national, provincial ou régional. Par contre, ces conditions varient selon la province où est située la communauté ou selon le type d'économie. Toutefois, outre des divergences 
quant à la conception et à l'utilisation des espaces de comparaison, la recherche quantitative sur les Amérindiens regorge également de variations dans la définition, et par extension dans la composition, des classes d'équivalence "Amérindiens » et Allochtones. Bien que le positionnement des Amérindiens et des non-Amérindiens dans un espace d'équivalence commun amène à penser ces catégories comme des entités homogènes en opposition l'une par rapport à l'autre, des fractures internes les traversent et celles-ci ne sont pas discutées, ou si peu, dans les analyses macrosociologiques nationales.

\section{OBJECTIF}

À la suite de la recension des écrits, il est apparu important de s'éloigner des analyses purement descriptives et comparatives entre les grandes «catégories» construites que sont les Autochtones et les Allochtones afin de pousser plus loin l'analyse des disparités socioéconomiques et de tenir compte de l'influence de conditions sociohistoriques de développement dissemblables au sein même des provinces sur les opportunités, entres autres sur le plan de l'emploi et de l'éducation. Il est apparu primordial de se centrer sur les dynamiques d'inégalités propres à une seule province en s'intéressant non seulement au degré d'isolement des collectivités, mais également à leur environnement immédiat.

Nous restreignons les analyses à une seule province, soit le Québec. Ce choix se justifie non seulement par la volonté de rendre compte de façon plus fine de l'hétérogénéité des situations vécues par les Amérindiens, mais également par le fait que les provinces interviennent différemment à l'égard des Autochtones via leurs politiques sociales ou la signature de conventions et la reconnaissance de droits particuliers (Dupuis, 1995).

Par ailleurs, les comparaisons intercollectivités actuelles n'ont pas permis de tenir compte du fait que les différences quant à la composition sociodémographique de ces collectivités, ne serait-ce que sur le plan de l'âge, pouvaient expliquer une partie des différences de moyennes. En conséquence, nous avons choisi d'analyser les données individuelles plutôt que d'utiliser des indices collectifs qui ne permettent pas de rendre compte de la variation des situations individuelles au sein des collectivités, ni de la composition différenciée de ces dernières. Les conditions socioéconomiques des individus se différencient selon divers facteurs tels le genre (Gerber, 1990), l'âge (Wotherspoon et Satzewich, 1993; Kulshreshtha et Dore, 2003; De Silva, 1999) et la scolarité (De Silva, 1999). Toute analyse des différences entre les groupes doit tenir compte des différences dans leur composition relativement à ces facteurs.

Nous centrons l'attention sur les résidants des communautés des Premières Nations. Nous comparons la situation socioéconomique de ces résidants à celle des résidants des communautés avoisinantes, ce qui permet de tenir compte de la similitude des contextes de vie tels le partage d'un marché de l'emploi similaire et la situation géographique, un facteur identifié comme déterminant pour expliquer les disparités entre Autochtones et Allochtones (McHardy et O’Sullivan, 2004; Armstrong, 
2001; White et Maxim, 2007; AINC, 1997), tout en isolant l'impact du fait de vivre sous le régime juridique propre aux réserves.

Enfin, les analyses portent sur les quatre dimensions de l'IBC, soit la scolarité, l'activité, le revenu et le logement, ce qui permet éventuellement de comparer nos résultats avec ceux des études précédentes. L’objectif est de s'éloigner de l'essentialisme attaché aux catégories «Amérindiens» et «non-Amérindiens» pour rendre compte de l'apport d'autres facteurs dans les dynamiques d'inégalité sociale. Pour cela, nous examinons sur quelles dimensions précisément et jusqu'à quel point l'autochtonité ainsi que la résidence dans une communauté des Premières Nations jouent un rôle dans les disparités socio-économiques existant entre résidants de communautés faisant face à un environnement immédiat, un emplacement géographique et un marché de l'emploi similaire, une fois les autres différences individuelles et communautaires contrôlées.

\section{MÉTHODOLOGIE}

\section{Population}

La population à l'étude comprend l'ensemble des individus qui, en 2001, résidaient soit dans une communauté des Premières Nations située sur le territoire du Québec, soit dans une collectivité avoisinante d'une telle communauté. La sélection des communautés considérées comme avoisinantes est faite en fonction d'un critère de proximité géographique, soit $25 \mathrm{~km}$ ou moins d'accès routier à une collectivité des Premières Nations. Deux exceptions ont été faites. Pour les réserves situées dans des régions isolées (Whapmagoostui, Wemotaci, etc.) ou qui ne sont pas reliées au réseau routier (Pakuashipi, Matimekosh/Lac John, etc.), les collectivités les plus près des réserves ont été identifiées comme avoisinantes et ce, peu importe la distance. Pour les communautés situées à proximité de grands centres urbains (Odanak et Wendake), seules les municipalités situées dans un rayon de $15 \mathrm{~km}$ ont été considérées comme avoisinantes.

Au total, 131 collectivités ( 35 des Premières Nations et 96 avoisinantes), réparties en 24 regroupements ${ }^{8}$, ont été retenues pour les analyses. Par ailleurs, pour les dimensions portant sur les caractéristiques individuelles, la population a été réduite à l'ensemble des personnes de 20 à 59 ans. Prendre les adultes de moins de 20 ans aurait donné un portrait biaisé de la scolarité puisque de plus en plus de jeunes finissent leurs études entre l'âge de 18 et de 20 ans. Enfin, comme de plus en plus de gens prennent leur retraite entre l'âge de 60 ans et 65 ans, garder cette population aurait biaisé les statistiques portant sur l'activité.

La population comprise est de 429850 individus (répartis dans 174625 ménages privés), dont 34575 demeurent dans l'une ou l'autre des collectivités des Premières Nations recensées ( 9050 ménages privés). La population des 20 à 59 ans est composée de 250170 personnes, dont 17215 vivant dans des collectivités amérindiennes.

8. La liste des regroupements avec leur composition est présentée en annexe. 
Suivant Wilkes (2010), seuls les répondants ayant déclaré une identité amérindienne, combinée ou non avec un statut ou avec une ascendance amérindienne, ont été identifiés comme Amérindiens. Dans la population retenue, près de $92 \%$ des individus n'ont déclaré aucune identité amérindienne, ascendance amérindienne ou statut d'Indien inscrit. Parmi les $8 \%$ restants, un peu plus de $88 \%$ s'identifient comme amérindiens, que ce soit uniquement $(2,7 \%)$, combiné avec une origine $(3,7 \%)$, à un statut $(2,7 \%)$ ou avec les deux $(79,2 \%)$. La restriction à la question d'identité exclut donc $12 \%$ de la population ayant donné une réponse positive à l'une ou l'autre des deux autres questions quantifiant l'autochtonité, soit ceux ayant déclaré une origine ou un statut d'Indien inscrit, mais non une identité. Parmi les répondants s'étant déclarés Amérindiens et résidant dans l'une ou l'autre des communautés à l'étude, un peu plus de $82 \%$ résident dans les collectivités des Premières Nations, près de $18 \%$ étant donc domiciliés dans les collectivités avoisinantes.

\section{Données}

Les données utilisées proviennent du micro-fichier de données confidentielles du recensement de 2001 issues du questionnaire long de Statistique Canada (Statistique Canada, 2001). Dans les collectivités des Premières Nations, tout comme dans les régions éloignées ou nordiques, le questionnaire long est utilisé pour tous les habitants. Dans le reste de la population, ce questionnaire est utilisé auprès d'un ménage sur cinq. Le recensement de 2001 a été préféré à celui de 2006 parce que le nombre de collectivités amérindiennes dénombrées ou avec des taux de réponse supérieurs à $75 \%$ y est plus grand et que certaines communautés ayant des caractéristiques particulières, dont la proximité d'un grand centre urbain, y ont participé. Notons toutefois qu'aucune communauté mohawk n’a été dénombrée depuis le recensement de 1981.

\section{Mesures}

\section{Les dimensions à l'étude}

Quatre dimensions sont investiguées, soit la scolarité, l'activité, le revenu et le logement. Les deux premières dimensions concernent les individus, alors que le revenu relève à la fois des individus et des ménages, et le logement, uniquement des ménages. Plusieurs indicateurs peuvent être utilisés pour bien refléter chacune des dimensions. Nous avons sélectionné ceux qui nous sont apparus les plus pertinents. Pour la scolarité, nous nous intéressons à l'alphabétisation fonctionnelle ( 9 années d'études complétées) et à la possession d'un diplôme d'études secondaires; pour l'activité, l'occupation du répondant (avoir été en emploi durant la période 2000-2001) et son statut d'emploi (c'est-à-dire travailleur majoritairement à temps plein ou à temps partiel) sont les indicateurs retenus; pour le revenu, la possession d'un revenu individuel supérieur au $75^{\mathrm{e}}$ centile de l'ensemble de la population à l'étude est analysée, ce qui permet de se distancier des recherches utilisant la moyenne sans accorder d'importance à l'étendue des variations dans le revenu. Finalement, les conditions de logement 
sont examinées par le biais de deux indicateurs, soit l'état du logement mesuré par le fait qu'il nécessite des réparations majeures et le surpeuplement mesuré par le fait qu'il $\mathrm{y}$ a plus d'une personne par pièce.

\section{Les facteurs explicatifs}

Les déterminants des inégalités retenus se répartissent entre des facteurs de niveau individuel (ou de ménages, le cas échéant) et des facteurs de contexte. Le nombre et la nature des facteurs individuels varient selon l'axe étudié. Deux variables indépendantes sont présentes dans l'ensemble des analyses de niveau individuel, soit l'âge (regroupé en 4 tranches de 10 ans de 20 à 59 ans) et le sexe, deux caractéristiques associées à la scolarité, à l'activité et au revenu des individus. Pour les analyses relatives à l'occupation, la scolarité - regroupée en quatre catégories, soit moins de 9 ans de scolarité, 9 ans à moins de 11 ans de scolarité, possession d'un diplôme d'études secondaires et fréquentation d'un établissement post-secondaire - est ajoutée. Pour celles relatives au revenu, la scolarité et l'occupation du répondant s'ajoutent. Une fois contrôlé l'apport de ces variables, le fait de s'identifier comme Amérindien est analysé pour chacun des indicateurs. Dans les analyses portant sur l'état du logement, seul le logarithme naturel du revenu total du ménage est utilisé comme variable explicative.

Pour ce qui est des facteurs de contexte, l'isolement des communautés et leur environnement immédiat sont d'abord analysés. Pour quantifier l'isolement, nous avons utilisé les zones géographiques établies par l'AINC, mesure utilisée également par White et Maxim (2007). Nous avons utilisé les mêmes critères pour classifier les collectivités avoisinant les communautés des Premières Nations. Une nouvelle catégorie a dû être ajoutée dans le cas où la communauté est un centre de services. L'effet de l'environnement immédiat est évalué par l'appartenance aux regroupements de communautés. La catégorisation en zones diffère des regroupements: près de $60 \%$ des regroupements (14 sur 24) sont composés de communautés ne partageant pas un accès routier semblable à un centre de services et ne se retrouvant donc pas classées dans la même zone. Finalement, l'effet résiduel attribuable à la résidence dans une collectivité des Premières Nations est étudié.

\section{Analyses}

La régression logistique avec entrée hiérarchique a été utilisée pour analyser l'impact des facteurs individuels et de contexte sur les divers indicateurs des dimensions à l'étude. Dans un premier temps, une régression ne contenant que les variables indépendantes de niveau individuel (ou des ménages, le cas échéant) a été réalisée. Par la suite, une seconde analyse a été effectuée en gardant uniquement les variables relatives aux communautés. Ceci a permis d'estimer la contribution relative de chacun des groupes de facteurs, excluant l'autochtonité et la résidence dans une collectivité des Premières Nations. Ensuite, une régression combinant variables individuelles (ou des ménages) avec variables contextuelles a été réalisée, ce qui informe sur la contribution totale des facteurs, toujours à l'exclusion de ceux relatifs à l'autochtonité et à la rési- 
dence dans une réserve. Enfin, les variables mesurant l'autochtonité (pour les analyses portant sur les individus) et la résidence dans une collectivité des Premières Nations ont été entrées de façon à évaluer leur apport supplémentaire unique à l'explication. Par souci de concision, seuls les modèles finaux et les informations sur les apports de variance aux diverses étapes sont présentés dans cet article. La contribution des variables à l'explication de la variance totale est évaluée par le pseudo- $\mathrm{R}^{2}$ de Nagelkerke. Ce coefficient a été préféré au $\mathrm{R}^{2}$ de Cox et Snell parce qu'il est corrigé pour se situer sur une échelle de -1 à +1 .

Les analyses sont effectuées sur des données pondérées de façon à se conformer aux normes de divulgation des données censitaires confidentielles. Afin de nous assurer de la validité des résultats, les analyses ont été également produites avec les données non pondérées. Aucune différence notable amenant à modifier l'interprétation n'est apparue. Par ailleurs, puisqu'il s'agit de résultats d'analyses réalisées, au moins partiellement, sur la population totale et non sur un échantillon, la significativité du test du Chi2 de Wald informe sur l'importance de la relation plutôt que sur la possibilité d'inférer la relation obtenue à une population.

\section{RÉSULTATS}

Les résultats relatifs aux caractéristiques individuelles - scolarité, activité, revenu individuel — sont présentés en premier lieu. Ils sont suivis de ceux du logement qui est une caractéristique de ménage. Pour chaque dimension, la présentation se fait en deux temps. Nous présentons d'abord la relation entre les variables explicatives et les variables dépendantes pour ensuite nous attarder à la part d'explication attribuable à chaque groupe de variables explicatives.

\section{La scolarité}

Dans un premier temps, nous nous intéressons à l'alphabétisation fonctionnelle. Les résultats présentés dans la première colonne du Tableau 1 indiquent que les chances de posséder une alphabétisation fonctionnelle sont nettement plus élevées chez les répondants plus jeunes ( 4,4 fois chez les 20-29 ans, 4,2 fois chez les 30-39 ans et 2,9 fois chez les 40-49 ans) que chez les 50-59 ans ${ }^{9}$, ce qui signifie qu'il y a eu une évolution positive avec le temps pour l'ensemble de la population. Par ailleurs, elles sont légèrement plus élevées ( 1,1 fois plus de chances) chez les femmes que chez les hommes.

9. Les résultats sont toujours interprétés en fonction de la catégorie de référence puisque le rapport de cote - $\exp (B)$ - présenté indique jusqu'à quel point les personnes de la catégorie considérée ont plus ou moins de chances d'avoir la caractéristique analysée (variable dépendante) que celles de la catégorie de référence. Ainsi, un $\exp (B)$ de 4,38 pour la catégorie des 20-29 ans indique que les répondants de ce groupe d'âge ont 4,4 fois plus de chances que les 50-59 ans (catégorie de référence) de posséder une $9^{\mathrm{e}}$ année de scolarité. Les chiffres sont arrondis à une décimale dans le texte pour faciliter la lecture. 
Tableau 1: Relation entre les facteurs explicatifs et les indicateurs de la situation socioéconomique

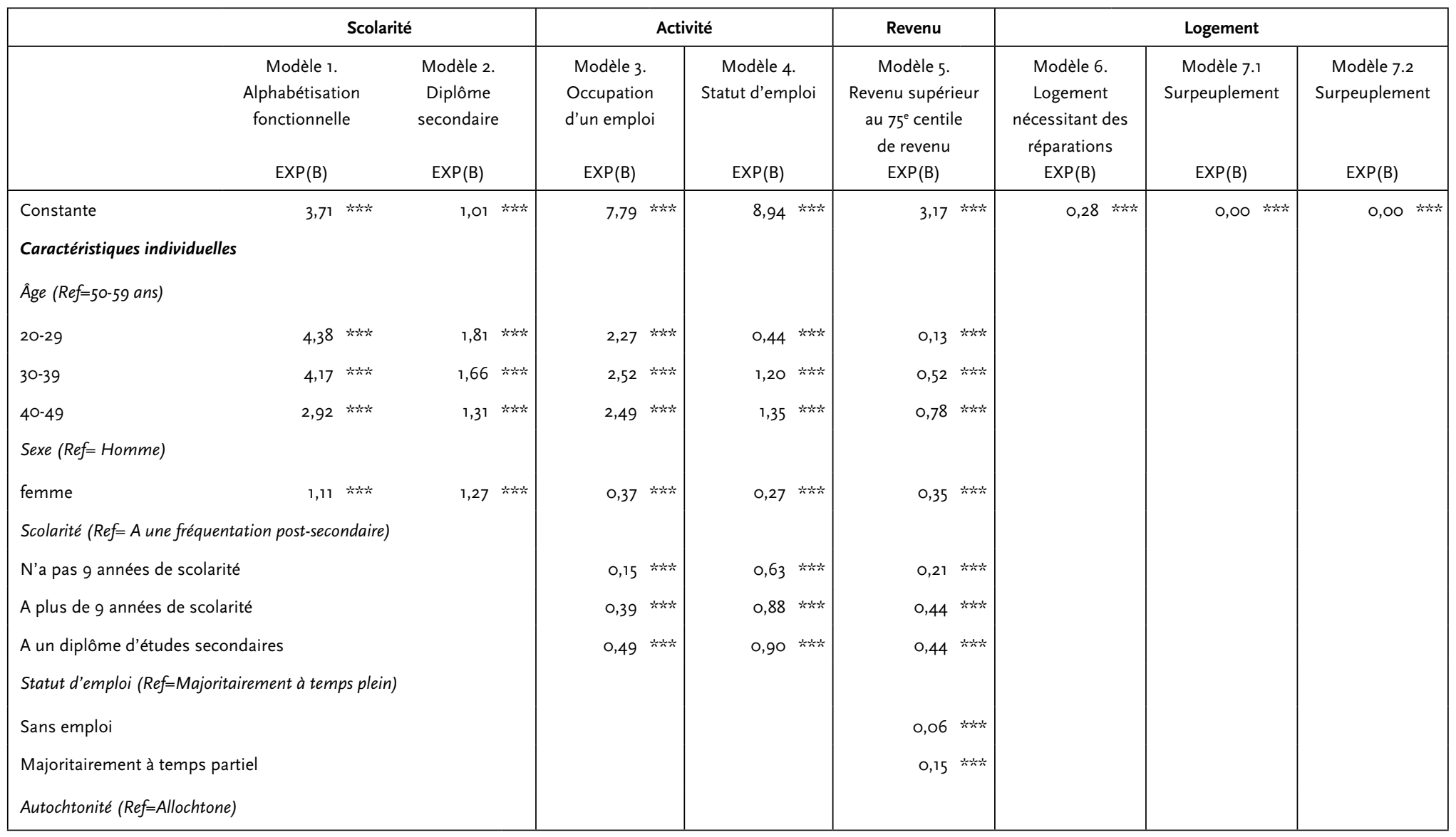




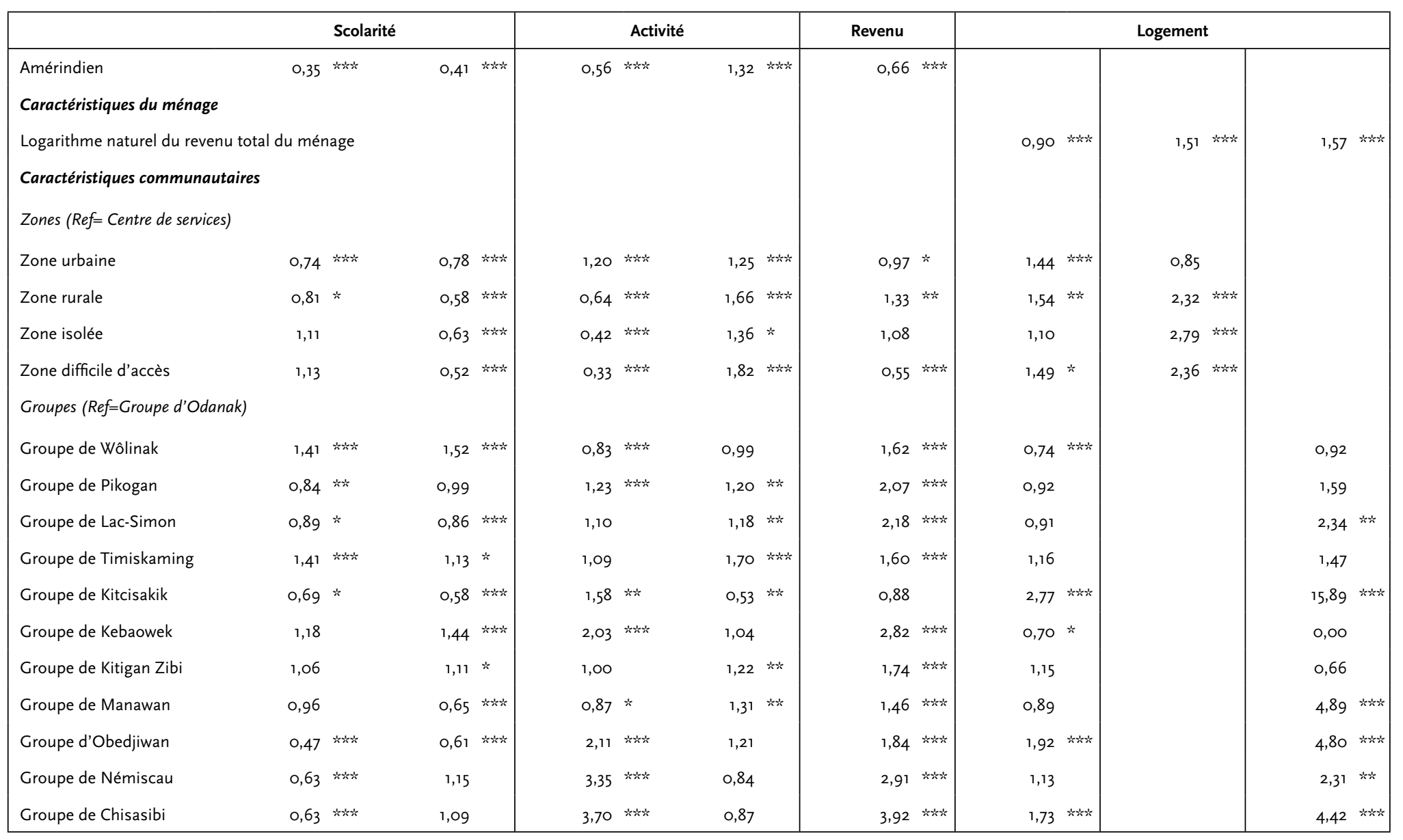




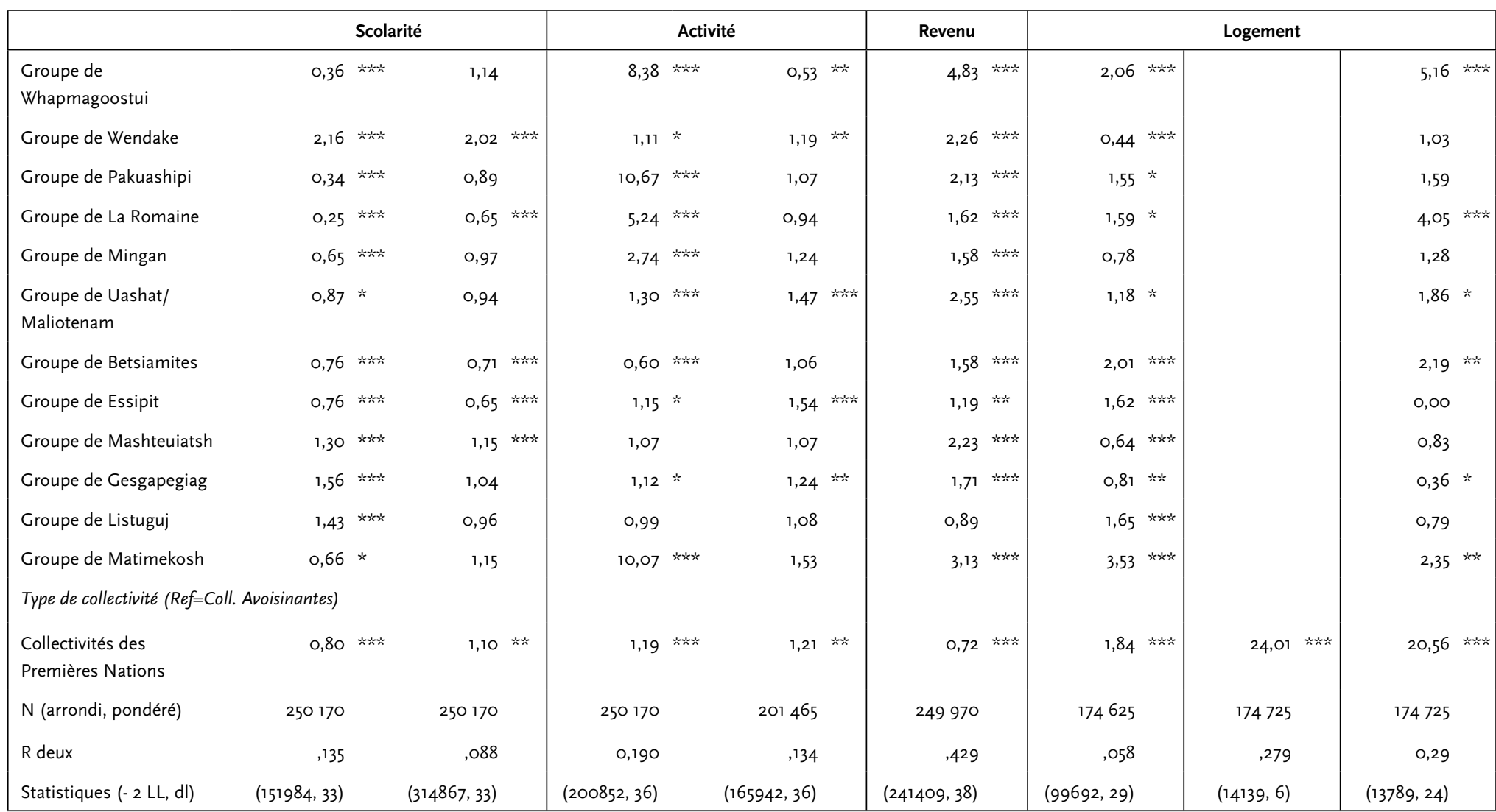

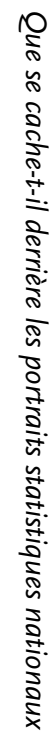

a. $*$ * $* * *, *$ indiquent une différence statistiquement significative aux seuils de $0,001,0,01$ et 0,05 respectivement. 
Pour ce qui est des facteurs contextuels, les résidants en zone rurale et urbaine ont respectivement $1,2^{10}$ et 1,3 fois moins de chances d'avoir terminé 9 années de scolarité que leurs homologues résidant dans un centre de services. L'accès à une alphabétisation fonctionnelle varie également en fonction du regroupement de résidences; les résidents du regroupement de Wendake, par exemple, ont des chances plus élevées d'avoir terminé 9 années de scolarité $(\exp (B)=2,16)$ que ceux du regroupement de référence (Odanak). Les chances les moins élevées se trouvent dans les regroupements de Whapmagoostui, Pakuashipi et La Romaine ${ }^{11}$ qui ont respectivement 2,8, 2,9 et 4,1 fois moins de chances d'avoir une alphabétisation fonctionnelle que les habitants des communautés composant le groupe d'Odanak.

Une fois contrôlés les facteurs individuels et contextuels, le fait de vivre dans une collectivité des Premières Nations constitue un léger désavantage, les résidants y ayant 1,3 fois moins de chances d'avoir une alphabétisation fonctionnelle. Par ailleurs, les personnes s'identifiant comme Amérindiennes ont 2,8 fois moins de chances que les non-Amérindiens d'avoir une alphabétisation fonctionnelle, toutes choses égales par ailleurs.

L'obtention d'un diplôme d'études secondaires est le deuxième indicateur de scolarisation. Tout comme pour l'alphabétisation fonctionnelle, les femmes sont légèrement plus susceptibles d'être diplômées du secondaire $(\exp (B)=1,27)$ que les hommes et les répondants qui ont moins de 50 ans ont plus de chances de détenir un diplôme d'études secondaires que les 50-59 ans. Toutefois, l'impact de l'âge est moins important que pour l'alphabétisation fonctionnelle.

Comparés aux résidants des centres de services, les habitants des quatre autres zones sont moins susceptibles d'être diplômés de l'école secondaire et ce, de 1,3 fois (zone urbaine) à 1,9 fois (zone difficile d'accès). Par ailleurs, à zone géographique identique, les chances de détenir un diplôme d'études secondaires varient selon les regroupements de collectivités. Par exemple, les personnes domiciliées dans une communauté constituant le groupe de Betsiamites bénéficient de 1,4 fois moins de chances de détenir un diplôme du secondaire que leurs homologues du groupe d'Odanak, bien que l'ensemble des collectivités de ces deux regroupements soient situées en zone urbaine. Enfin, une fois contrôlés les facteurs individuels et de contexte, les résidants des communautés des Premières Nations ont $1,1(\mathrm{p}<0,001)$ fois plus de chances que ceux des collectivités avoisinantes de détenir un diplôme d'études secondaires. Par contre, les personnes s'identifiant comme Amérindiennes ont 2,4 fois moins de chances que les non-Amérindiens d'avoir décroché un tel diplôme. Les Amérindiens vivant

10. Afin de faciliter la lecture, les coefficients négatifs — plus petits que 1 — ont été ramenés sur une base comparative commune aux coefficients positifs en utilisant la formule 1/exp(B). Par exemple, pour un $\exp (B)$ de la catégorie zone rurale égal à $0,84,1 / \exp (B)$ donne 1,23. Les résidants des communautés en zone rurale ont donc 1,23 fois moins de chances que ceux des centres de services (catégorie de référence) d'avoir une scolarité fonctionnelle (variable dépendante), ceci toutes choses égales par ailleurs.

11. Les noms de communauté qui avaient cours en 2001, au moment où les données ont été recueillies par Statistique Canada, sont utilisés dans le texte. Certains noms ont été changés depuis. 
dans les collectivités des Premières Nations seraient donc légèrement avantagés par rapport à ceux vivant dans les collectivités avoisinantes.

Le fait qu'il y a des relations, même fortes, entre les variables explicatives et les variables dépendantes ne donne pas d'indication sur l'importance de la contribution des facteurs explicatifs à la variation de la variable à expliquer. Le tableau 2 présente cette information pour chacune des variables dépendantes en fonction des groupes de variables explicatives.

Pour ce qui est de la scolarité, les facteurs individuels, soit l'âge et le sexe, expliquent à peine plus de $5 \%$ de la variation dans l'alphabétisation fonctionnelle et à peine plus de $1 \%$ de la variation dans l'acquisition d'un diplôme d'études secondaires. Les facteurs de contexte apparaissent donc d'autant plus importants. Autant pour l'alphabétisation fonctionnelle que pour la possession d'un diplôme d'études secondaires, ce sont l'isolement et l'environnement immédiat qui sont les facteurs déterminants dans l'explication de l'accès à la scolarité. Pour ce qui est de l'alphabétisation fonctionnelle, la zone de résidence explique $3,2 \%$ de la variance et les regroupements $3 \%$, pour un total de 6,2\% de la variance expliquée par les facteurs contextuels. Quant à la possession d'un diplôme d'études secondaires, la contribution des zones est de $2 \%$ et celle des regroupements de 3,7\%, pour un total de $6,5 \%$.

Quel est, au-delà de ces facteurs, l'apport du fait de résider dans une communauté des Premières Nations et d'être amérindien? Ce fait explique 1,5\% de la variance dans l'alphabétisation fonctionnelle et $0,5 \%$ de la variance dans l'acquisition d'un diplôme d'études secondaires. Au-delà du lieu de résidence, l'autochtonité joue un rôle encore plus mineur. Une fois contrôlées les autres caractéristiques, celle-ci contribue à l'explication d'environ un demi-point de pourcentage de la variance totale, que ce soit pour l'alphabétisation fonctionnelle ou la possession d'un diplôme d'études secondaires. Au total, les facteurs d'autochtonité et de résidence dans une collectivité des Premières Nations expliquent $2 \%$ sur une variance expliquée totale de $13,5 \%$ pour ce qui est de l'alphabétisation fonctionnelle et moins de $1 \%$ sur un total de $8,8 \%$ pour ce qui est de l'acquisition d'un diplôme d'études secondaires. C'est donc d'abord et avant tout l'emplacement géographique et l'environnement immédiat des résidants de certaines collectivités des Premières Nations qui les désavantagent, ce qui est aussi vrai pour les résidants des communautés avoisinantes situées dans les mêmes régions.

\section{L'activité}

Les analyses montrent que l'âge est lié à des chances inégales d'accéder à un emploi: les travailleurs de moins de 50 ans ont tous plus de chances (de 2,3 à 2,5 fois) d'avoir occupé un emploi en 2000-2001 que les 50-59 ans. Par ailleurs, les femmes apparaissent 2,7 fois moins susceptibles $(\exp (B)=0,37)$ que les hommes d'avoir occupé un emploi rémunéré durant la période. La scolarité apparaît comme déterminante dans les probabilités d'occuper un emploi: les répondants qui ont acquis un diplôme d'études secondaires ont 2 fois moins de chances d'occuper un emploi que ceux qui ont fréquenté un établissement post-secondaire comparativement à 2,5 fois moins de 


\begin{tabular}{|c|c|c|c|c|c|c|c|c|c|c|c|}
\hline \multirow{2}{*}{$\begin{array}{l}\text { Modèle } \\
\text { de départ }\end{array}$} & \multirow{2}{*}{$\begin{array}{l}\text { Facteurs } \\
\text { introduits } \\
\text { successivement } \\
\text { dans le modèle } \\
\text { de départ }\end{array}$} & \multicolumn{2}{|c|}{$\begin{array}{c}\text { Alphabétisation } \\
\text { fonctionnelle (modèle 1) }\end{array}$} & \multicolumn{2}{|c|}{$\begin{array}{l}\text { Diplôme secondaire } \\
\text { (modèle 2) }\end{array}$} & \multicolumn{2}{|c|}{$\begin{array}{l}\text { Occupation d'un emploi; } \\
\text { période de } 2000 \text { à } 2001 \\
\text { (modèle 3) }\end{array}$} & \multicolumn{2}{|c|}{$\begin{array}{l}\text { Occupation d'un emploi } \\
\text { majoritairement à temps } \\
\text { plein (modèle 4) }\end{array}$} & \multicolumn{2}{|c|}{$\begin{array}{c}\text { Revenu supérieur individuel } \\
\text { au } 75^{\mathrm{e}} \text { centile } \\
\text { (modèle 5) }\end{array}$} \\
\hline & & $\begin{array}{c}\% \text { de } \\
\text { variance } \\
\text { totale } \\
\text { expliquée }\end{array}$ & $\begin{array}{l}\text { \% ajouté de } \\
\text { la variance } \\
\text { expliquée }\end{array}$ & $\begin{array}{c}\% \text { de } \\
\text { variance } \\
\text { totale } \\
\text { expliquée }\end{array}$ & $\begin{array}{l}\text { \% ajouté de } \\
\text { la variance } \\
\text { expliquée }\end{array}$ & $\begin{array}{c}\% \text { de } \\
\text { variance } \\
\text { totale } \\
\text { expliquée }\end{array}$ & $\begin{array}{l}\text { \% ajouté de } \\
\text { la variance } \\
\text { expliquée }\end{array}$ & $\begin{array}{c}\% \text { de } \\
\text { variance } \\
\text { totale } \\
\text { expliquée }\end{array}$ & $\begin{array}{l}\text { \% ajouté de } \\
\text { la variance } \\
\text { expliquée }\end{array}$ & $\begin{array}{c}\% \text { de } \\
\text { variance } \\
\text { totale } \\
\text { expliquée }\end{array}$ & $\begin{array}{c}\% \text { ajouté de } \\
\text { la variance } \\
\text { expliquée }\end{array}$ \\
\hline \multirow{5}{*}{$\begin{array}{l}\text { Facteurs } \\
\text { individuels } \\
\text { (sans } \\
\text { autochtonité) }\end{array}$} & & $5,3 \%$ & & $1,4 \%$ & & $17,8 \%$ & & $12,6 \%$ & & $41,7 \%$ & \\
\hline & $\begin{array}{l}\text { Zones } \\
\text { géographiques }\end{array}$ & $8,5 \%$ & $3,2 \%$ & $4,2 \%$ & $2,8 \%$ & $18,0 \%$ & $0,2 \%$ & $12,9 \%$ & $0,3 \%$ & $41,8 \%$ & $0,1 \%$ \\
\hline & $\begin{array}{l}\text { Regroupements } \\
\text { de collectivités }\end{array}$ & $11,5 \%$ & $3,0 \%$ & $7,9 \%$ & $3,7 \%$ & $18,8 \%$ & o,8\% & $13,2 \%$ & $0,3 \%$ & $42,6 \%$ & o,8\% \\
\hline & $\begin{array}{l}\text { Types de } \\
\text { collectivités }\end{array}$ & $13,0 \%$ & $1,5 \%$ & $8,4 \%$ & $0,5 \%$ & $18,9 \%$ & $0,1 \%$ & $13,4 \%$ & $0,2 \%$ & $42,8 \%$ & $0,2 \%$ \\
\hline & $\begin{array}{l}\text { Autochtonité } \\
\text { (modèle } \\
\text { parcimonieux) }\end{array}$ & $13,5 \%$ & $0,5 \%$ & $8,8 \%$ & $0,4 \%$ & $19,0 \%$ & $0,1 \%$ & $13,4 \%$ & ০,०\% & $42,9 \%$ & $0,1 \%$ \\
\hline \multicolumn{2}{|c|}{$\begin{array}{l}\text { Modèle final facteurs individuels } \\
\text { seulement }\end{array}$} & $11,0 \%$ & & $5,2 \%$ & & $18,0 \%$ & & $12,8 \%$ & & $42,0 \%$ & \\
\hline \multicolumn{2}{|c|}{$\begin{array}{l}\text { Modèle final facteurs } \\
\text { communautaires seulement }\end{array}$} & $6,6 \%$ & & $6,7 \%$ & & $1,5 \%$ & & 0,8\% & & $2,5 \%$ & \\
\hline
\end{tabular}


chances pour ceux qui ont acquis seulement une alphabétisation fonctionnelle et 6,8 fois moins de chances pour ceux qui n'ont pas terminé une $9^{e}$ année.

Pour ce qui est des facteurs de contexte, les résidants en zone urbaine sont les seuls à être plus susceptibles $(\exp (B)=1,2)$ que les résidants des centres de services d'avoir occupé un emploi, les citoyens des autres zones étant significativement défavorisés (de 1,6 à 3 fois moins de chances) lorsque comparés à leurs homologues des centres de services. De plus, l'effet de l'environnement immédiat sur l'accès à l'emploi est apparent; les chances d'être en emploi sont 10,1 et 10,7 fois plus élevées pour les résidants des regroupements de Pakuashipi et de Matimekosh, respectivement, que pour leurs homologues du regroupement d'Odanak, alors qu'elles sont moindres dans trois regroupements uniquement. Il est possible que l'on assiste ici à un effet inversé, soit que, en région éloignée ou isolée, les personnes qui n'ont pas d'emploi et peu d'espoir d'en trouver un aient tendance à s'exiler vers les régions urbaines et que celles qui immigrent dans ces régions le fassent surtout parce qu'elles y ont trouvé un emploi. Enfin, toutes choses égales par ailleurs, les résidants des collectivités des Premières Nations ont 1,2 fois plus de chances que ceux des collectivités avoisinantes d'avoir occupé un emploi durant la période 2000-2001. Par contre, les Amérindiens ont 1,8 fois moins de chances que les non-Amérindiens d'avoir occupé un emploi durant cette période ${ }^{12}$.

Nous nous attardons maintenant aux facteurs liés au fait d'occuper un emploi à temps plein plutôt qu'à temps partiel chez les personnes en emploi. Les analyses montrent que les travailleurs âgés de 20-29 ans ont plus de deux fois moins de chances $(\exp (B)$ de 0,44$)$ que les 50-59 ans d'avoir travaillé majoritairement à temps plein, alors que les travailleurs de 30 à 49 ans sont, quant à eux, légèrement favorisés (1,2 et 1,3 fois plus de chances selon les groupes d'âge) par rapport à ceux-ci. Par ailleurs, les femmes sont nettement moins susceptibles (3,7 fois moins) que les hommes d'avoir occupé un emploi à temps plein. La scolarité est également un facteur déterminant: plus les travailleurs sont scolarisés, plus leurs chances d'avoir occupé un emploi à temps plein sont élevées. Toutefois, cet impact est moins prononcé que pour le fait d'avoir occupé un emploi.

Les travailleurs des quatre zones (urbaine, rurale, isolée et difficile d'accès) ont significativement un peu plus de chances que ceux des centres de services d'avoir occupé un emploi à temps plein, et ce, dans un intervalle de 1,1 à 1,6 fois. L'environnement immédiat contribue également à l'explication des variations: les chances d'occuper un emploi à temps plein sont significativement plus élevées pour les résidants de neuf regroupements que pour ceux du regroupement d'Odanak d'occuper un emploi à temps plein alors que celles de deux regroupements Timiskaming et Whapmaghoostui — sont significativement moins élevées.

12. L'introduction de la variable d'identité rend positive la relation observée entre l'emploi et la résidence dans une collectivité des Premières Nations, ce qui signifierait que, hors des collectivités des Premières Nations, les Amérindiens ont moins de chance d'être en emploi alors que dans les collectivités, ils ont plus de chances d'être en emploi. 
Pour cet indicateur, la résidence dans une collectivité des Premières Nations plutôt que dans une collectivité avoisinante est associée à des chances plus élevées pour les travailleurs d'être à temps plein $(\exp (B)=1,26)$. Contrairement à ce qui a été constaté pour l'accès à un emploi, les Amérindiens ont 1,3 fois plus de chances que les travailleurs non Amérindiens d'avoir travaillé majoritairement à temps plein, toutes choses égales par ailleurs.

En ce qui concerne l'activité, tant pour l'occupation d'un emploi que le statut de cet emploi, la contribution des facteurs individuels inclut maintenant la scolarité. Le tableau 2 montre que les facteurs individuels expliquent près de $18 \%$ de la variation dans l'occupation d'un emploi et près de $13 \%$ de la variation dans l'accès à un emploi à temps plein. La scolarité apparaît comme le principal facteur lié à l'accès à un emploi, alors que le sexe et l'âge sont les principaux contributeurs pour le statut d'emploi.

La contribution des facteurs collectifs s'avère alors minime lorsque comparée à celle des facteurs individuels. La zone de résidence explique à peine $0,2 \%$ de la variation dans l'occupation d'un emploi et $0,3 \%$ de la variation dans le statut d'emploi. $\mathrm{Au}$-delà, les regroupements expliquent $0,8 \%$ de la variation dans l'accès à un emploi et $0,3 \%$ de la variation dans le statut.

Le fait de résider dans une collectivité des Premières Nations ajoute très peu à l'explication de la variance, soit $0,2 \%$ de variance expliquée pour l'accès à un emploi et $0,1 \%$ pour le statut d'emploi. L'autochtonité quant à elle ne contribue pas à l'explication de la variance, une fois les autres caractéristiques contrôlées. Au total, 19\% de la variance totale est expliquée pour l'accès à l'emploi et 13,4\% pour le statut d'emploi.

\section{Le revenu}

Les analyses montrent que l'âge est corrélé à des chances différenciées de posséder un revenu individuel élevé, soit un revenu qui se situe au-delà du $75^{\mathrm{e}}$ centile de la distribution des revenus de la population retenue: les 20-29 ans ont 7,8 fois moins de chances d'avoir un tel revenu que les 50-59 ans. Ces chances sont aussi moindres chez les 30-39 ans (1,9 fois) et les 40-49 ans (1,3 fois). Les femmes sont également moins susceptibles ( 2,8 fois) que les hommes d'avoir un revenu élevé.

La scolarité est associée à des chances différenciées de posséder un revenu élevé: ceux ayant fréquenté un établissement postsecondaire obtiennent les chances les plus élevées alors que ceux qui n'ont pas une alphabétisation fonctionnelle sont les plus désavantagés. Sans trop de surprises, les travailleurs à temps plein obtiennent les meilleures chances de posséder un revenu individuel élevé alors que les travailleurs à temps partiel obtiennent des chances 6,8 fois moindres que ces derniers et les personnes sans emploi 15,6 fois moindres.

Pour ce qui est du contexte, les analyses montrent que seuls les résidants en zone rurale présentent des chances plus élevées que ceux des centres de services d'avoir un revenu supérieur, les résidants en zones urbaine (1,03 fois moins) et difficile d'accès ( 1,8 fois moins) étant désavantagés par rapport à ces derniers. À zone et à caractéristiques individuelles similaires, les résidants de presque tous les regroupements de 
collectivités sont plus susceptibles de bénéficier d'un revenu élevé que ceux du regroupement d'Odanak. Seuls les résidents des regroupements de Kitcisakik et de Listuguj font exception en ne se différenciant pas de ceux du regroupement d'Odanak.

Enfin, les résidants des collectivités des Premières Nations ont 1,4 fois moins de chances que ceux des collectivités avoisinantes d'avoir un revenu supérieur. La variable d'autochtonité demeure significative lorsque toutes les autres caractéristiques sont contrôlées: les Amérindiens sont 1,5 fois moins susceptibles que les non-Amérindiens de posséder un revenu élevé.

Pour ce qui est de l'accès à un revenu supérieur, les inégalités de revenu sont principalement expliquées par les caractéristiques individuelles. Le tableau 2 montre que près de $42 \%$ de la variance s'explique par celles-ci sur un total de $43 \%$ de variance expliquée. La scolarité et le fait d'occuper un emploi, encore plus à temps plein, sont déterminants. Au-delà, les variables collectives expliquent moins de $1 \%$ de la variance totale. Le fait de résider dans une collectivité amérindienne ajoute à peine $0,2 \%$ à l'explication. En ce qui concerne l'autochtonité, bien qu'elle soit corrélée aux inégalités de revenu, elle joue également un rôle très mineur au-delà des différences relatives, entre autres à l'accès à la scolarité.

\section{Le logement}

L'analyse montre que le revenu familial, transformé en son logarithme, est significativement et négativement lié à la probabilité de demeurer dans un logement requérant des rénovations majeures $(\exp (B)=0,9)$. Par ailleurs, les conditions de logement s'altèrent en dehors des centres de services; les chances d'habiter dans une demeure nécessitant des réparations majeures étant plus élevées pour l'ensemble des autres zones, à l'exception de la zone isolée ${ }^{13}$, et ce, de 1,4 à 1,5 fois. Pour ce qui est des regroupements de collectivités, les résidants de 11 regroupements obtiennent des chances de 1,2 à 3,5 fois plus élevées d'habiter un logement en mauvaise condition que ceux du regroupement d'Odanak, les regroupements de Kitcisakik et de Matimekosh étant les plus problématiques, tandis que ceux de cinq regroupements bénéficient de chances de 1,2 à 2,3 fois moindres.

Enfin, toutes choses égales par ailleurs, les résidants des collectivités des Premières Nations ont 1,8 fois plus de chances que ceux des collectivités avoisinantes de demeurer dans un logement en mauvais état.

La résidence dans un logement comptant plus d'une personne par pièce est le deuxième indicateur de l'état des logements habitués. Les variables de zones et de regroupements ne peuvent pas être entrées conjointement dans cette analyse puisqu'il existe un problème de multicolinéarité. Pour cette raison, le modèle 7.1 présente l'effet combiné des variables de revenu du ménage, de zone et de type de collectivité tandis que le modèle 7.2 présente celui des variables de revenu du ménage, de regroupement et de type de collectivité.

13. La catégorie n'est plus significative lorsque le type de communauté habitée est pris en compte. 
Le revenu total du ménage, toujours transformé en son logarithme naturel, est positivement et significativement lié à la probabilité de vivre dans un logement surpeuplé dans les deux modèles de régression. Ceci signifie que le surpeuplement n'est pas dû à un manque de revenu. Au contraire, il faut conclure que, dans les logements où il y a plus de personnes, il y a également plus de personnes qui ont des revenus. L'isolement de la collectivité est lié à des chances inégales de vivre dans un logement surpeuplé, ces chances étant environ 2,3 fois supérieures en zone rurale et en zone difficile d'accès et 2,8 fois supérieures en zone isolée que dans les centres de services. L'environnement immédiat contribue également à l'explication des inégalités de logement. Comparés aux résidants du groupement d'Odanak, ceux de onze regroupements présentent des chances plus élevées de résider dans un logement surpeuplé, et ce, dans un intervalle de 1,9 à 15,9 fois, alors que seuls les résidants du regroupement de Gesgapegiag ont des chances significativement moindres de demeurer dans un logement surpeuplé. Pour les deux modèles, la résidence dans une collectivité des Premières Nations plutôt que dans une collectivité avoisinante est associée à des chances beaucoup plus grandes de vivre dans un logement surpeuplé, et ce, de 20,6 à 24 fois. Ainsi, le type de communauté de résidence apparaît être le facteur le plus déterminant pour ce qui est du surpeuplement des logements. À titre d'exemple, en 2001, la proportion de logements comprenant plus d'une personne par pièce était de $46 \%$ dans les collectivités des Premières Nations du regroupement de Kitcisakik, 28,6\% à Manawan, 27,3\% à Lac-Simon et 19,5\% dans les collectivités du regroupement de La Romaine, cette situation étant rare dans les collectivités avoisinantes ${ }^{14}$.

Les informations relatives à la variance expliquée dans les conditions de logement sont présentées dans le tableau 3. Le revenu du ménage joue un rôle infime dans l'explication des disparités de conditions de logement, expliquant tout au plus $1 \%$ de la variance. Les zones géographiques et les regroupements expliquent respectivement $1,8 \%$ et 3,4\% de la variance dans le fait d'habiter un logement nécessitant des réparations majeures alors que le fait de résider dans une collectivité amérindienne explique $3,8 \%$ de la variation. Pour ce qui est du surpeuplement, les variables de zones et de regroupements partagent une telle part de variance qu'il est impossible de les garder toutes les deux dans le même modèle. Les zones expliquent un peu plus de $13 \%$ de variance unique alors que les regroupements en expliquent $19 \%$. Le fait de résider dans une communauté des Premières Nations ajoute près de $14 \%$ à l'explication audelà de la variable de zone et près de $10 \%$ au-delà de la variable de regroupement, soit au minimum un tiers de l'ensemble de la variation expliquée puisque, au total, environ $30 \%$ de la variance dans le surpeuplement des logements est expliquée. La résidence dans une collectivité amérindienne est donc un facteur majeur d'explication pour ce qui est du surpeuplement.

14. Des situations de surpeuplement dans les collectivités avoisinantes sont notables dans six regroupements. Cependant, la proportion de logements surpeuplés dans ces cas ne dépasse le seuil de $1 \%$ que dans trois regroupements, ceux de Kitcisakik (2,7\%), de Matimekosh (4,7\%) et de Whapmagoostui (14,2\%). 
Tableau 3: Contribution à l'explication de la variance — Logement

\begin{tabular}{|c|c|c|c|c|c|c|c|}
\hline \multirow[b]{2}{*}{ Modèle de départ } & \multirow[b]{2}{*}{$\begin{array}{l}\text { Facteurs introduits successivement } \\
\text { dans le modèle de départ }\end{array}$} & \multicolumn{2}{|c|}{ État du logement (modèle 6) } & \multicolumn{2}{|c|}{ Surpeuplement (modèle 7.1) } & \multicolumn{2}{|c|}{ Surpeuplement (modèle 7.2) } \\
\hline & & $\begin{array}{l}\% \text { de variance } \\
\text { totale expliquée }\end{array}$ & $\begin{array}{c}\text { \% ajouté de la } \\
\text { variance expliquée }\end{array}$ & $\begin{array}{l}\text { \% de variance } \\
\text { totale expliquée }\end{array}$ & $\begin{array}{c}\text { \% ajouté de la } \\
\text { variance expliquée }\end{array}$ & $\begin{array}{l}\text { \% de variance } \\
\text { totale expliquée }\end{array}$ & $\begin{array}{c}\text { \% ajouté de la } \\
\text { variance expliquée }\end{array}$ \\
\hline \multirow[t]{3}{*}{ Facteurs familiaux } & & $0,2 \%$ & & $1,0 \%$ & & $1,0 \%$ & \\
\hline & Zones géographiques & $2,0 \%$ & $1,8 \%$ & & & & \\
\hline & Regroupements de collectivités & $5,4 \%$ & $3,4 \%$ & & & & \\
\hline \multirow{3}{*}{$\begin{array}{l}\text { Zones géographiques } \\
\text { Regroupements de } \\
\text { collectivités }\end{array}$} & & & & $14,2 \%$ & $13,2 \%$ & & \\
\hline & & & & & & $20,0 \%$ & $19,0 \%$ \\
\hline & Types de collectivités & $5,8 \%$ & $0,4 \%$ & $27,9 \%$ & $13,7 \%$ & $29,8 \%$ & $9,8 \%$ \\
\hline
\end{tabular}




\section{DISCUSSION}

Pour les dimensions relatives aux individus, la scolarité apparaît centrale dans l'explication des inégalités, tant pour l'accès à un emploi que pour l'accès à un revenu supérieur. L'accès à cette scolarité est, quant à lui, plus lié à des facteurs contextuels qu'individuels.

Les résultats présentés montrent que l'impact du fait d'être amérindien ou de résider dans une collectivité des Premières Nations existe même en présence d'autres facteurs explicatifs. Toutefois, cet impact varie énormément d'un indicateur à l'autre et, une fois contrôlés les facteurs individuels et collectifs pertinents, le fait de vivre dans une collectivité des Premières Nations ou d'être amérindien n'est pas toujours négatif.

\section{L'impact des facteurs individuels communs}

Les facteurs tels que l'âge et le genre contribuent d'abord et avant tout à l'explication des inégalités scolaires. Les résultats montrent une évolution positive dans l'accès à la scolarité avec le temps puisque les plus jeunes sont plus scolarisés. Ils montrent également que l'accès à la scolarité est central dans l'explication des inégalités d'emploi et de revenu, ce qui permet de mettre en perspective les analyses descriptives relatives aux écarts salariaux et d'emploi entre Autochtones et Allochtones, en rendant manifeste l'importance de l'accès à la scolarité comme principal facteur explicatif.

Les résultats montrent que les travailleurs de 30 à 49 ans sont plus susceptibles d'être en emploi et d'occuper un emploi à temps plein que les travailleurs plus jeunes ou plus âgés, ce qui confirme les travaux de Cheal (2003). Par ailleurs, le genre des répondants est lié à des chances différenciées, les hommes étant plus fréquemment en emploi à scolarité égale. Les résultats pour ce qui est des déterminants du revenu sont cohérents avec les travaux de De Silva (1999) pour qui la scolarité et l'âge sont les principaux déterminants du revenu chez les travailleurs, amérindiens comme non amérindiens.

\section{L'impact des facteurs de contexte communs}

Pour toutes les analyses, à l'exception de celles ayant trait à la scolarité, les facteurs de contexte communs sont apparus secondaires dans l'explication des inégalités. Les caractéristiques individuelles sont dominantes dans l'explication de la situation socioéconomique, ce qui est cohérent avec les résultats de recherche pancanadiens de l'AINC (1997).

L'impact du facteur d'isolement est conforme à ce qui était attendu. La zone habitée est liée à des chances inégales de se scolariser: résider loin d'un centre de services est lié à une détérioration des chances de posséder une alphabétisation fonctionnelle ou un diplôme d'études secondaires, peu importe le type de collectivité habitée, et ce, même en prenant en compte les facteurs individuels. Ces résultats sont conformes à ceux présentés par White et Maxim (2007). 
L'existence d'une relation entre la zone habitée et l'emploi est aussi confirmée par les analyses présentées. Contrairement à ce que laissaient croire les analyses sur l'IBC activité (White et Maxim, 2007), la résidence en zone isolée n'offre pas des chances d'emploi équivalentes à la résidence en zone urbaine. Lorsque les facteurs pertinents sont contrôlés, les probabilités d'être en emploi s'amoindrissent avec l'isolement, ce qui est cohérent avec les données présentées par l'AINC (1997). Toutefois, les regroupements composés uniquement de communautés situées en zone difficile d'accès offrent tous des chances plus grandes d'être en emploi que ceux composés uniquement de collectivités en zone urbaine, laissant croire que les collectivités éloignées ayant un environnement immédiat que l'on pourrait qualifier d'homogène (uniquement des collectivités qui ne sont pas liées au réseau routier) offrent des opportunités d'emploi différentes de celles dont l'environnement immédiat est plus hétérogène (composé de communautés en zone difficile d'accès et de différentes autres zones). Les données ne permettent pas d'affirmer avec certitude que ces disparités s'expliquent par le type d'économie prédominante tel que suggéré par Duhaime et Godmaire (2002), mais il s'agit d'une piste d'explication plausible. Rappelons que les aires allochtones d'économie à grande échelle sont caractérisées par une population issue d'une immigration récente. La migration vers ces aires est principalement motivée par une forte demande en travailleurs qualifiés et des opportunités d'emploi singulières, notamment en ce qui a trait aux salaires offerts (Duhaime et Godmaire, 2002).

Pour ce qui est du revenu, l'influence de la zone est moins marquée, c'est-à-dire que les chances d'avoir un revenu élevé ne se différencient pas pour toutes les zones. Les résultats ne concordent pas avec ceux présentés par Maxim et White (2007) pour l'IBC revenu. Les chances d'avoir un revenu élevé sont à leur valeur la plus élevée en zone rurale plutôt qu'en zone urbaine ou isolée. Les différences pourraient s'expliquer par le fait que nos résultats sont contrôlés pour l'accès à la scolarité et à un emploi, mais également par un indicateur différent, soit le $75^{\mathrm{e}}$ centile du revenu dans notre cas et le revenu par personne pour Maxim et White (2007).

La relation entre l'isolement et la qualité des logements ou leur surpeuplement est médiatisée par le type de communauté. Les logements surpeuplés sont plus nombreux dès que l'on s'éloigne des centres de services et de leur périphérie urbaine, mais cela est nettement plus marqué dans les collectivités des Premières Nations.

Les résultats permettent de constater que l'environnement immédiat des communautés influence les opportunités scolaires, d'emploi, de revenu et de logement de leurs résidants, au-delà des différences liées à la zone géographique. Cet indicateur englobe des facteurs plus spécifiques comme la situation générale du marché du travail, les modèles de travail (types d'industrie, entre autres) et la présence d'infrastructures (écoles primaires et secondaires, en particulier) à proximité du lieu de résidence. Dans une certaine mesure, l'indicateur d'environnement immédiat utilisé dans cette étude s'apparente à l'indicateur de type d'échelle d'économie utilisé par Duhaime et Godmaire (2002) ou à celui de région discuté par Southcott (2003). L'environnement immédiat permet d'expliquer une plus large part de variance unique que la zone, ce 
qui confirme la pertinence du choix de restreindre la population à l'étude. Toutefois, en ce qui concerne les conditions de logement, les indicateurs d'environnement immédiat et d'isolement sont fortement liés de sorte qu'il est difficile d'identifier l'apport unique de chaque facteur.

\section{L'impact de l'autochtonité et de la résidence dans une communauté des Premières Nations}

De façon générale, la résidence dans une collectivité des Premières Nations est associée à une moins grande probabilité d'avoir accès à une alphabétisation fonctionnelle et d'avoir un revenu élevé à scolarité et activité égales. Elle est également fortement associée à une plus grande probabilité de vivre dans un logement en mauvaise condition et surtout surpeuplé, ce qui confirme les travaux d'autres auteurs (McHardy et O’Sullivan, 2004; O’Sullivan et McHardy, 2004; AINC, 1997) et ce, même en tenant compte de l'isolement et de l'environnement immédiat.

Par contre, les chances de posséder un diplôme d'études secondaires sont plus élevées dans les communautés des Premières Nations, une fois contrôlé l'ensemble des facteurs individuels et contextuels pertinents. Nos résultats diffèrent des recherches recensées en ce qui concerne l'emploi. Contrairement à ce qu'avançaient McHardy et O'Sullivan (2004) et O'Sullivan et McHardy (2004) pour l'ensemble du Canada, nos analyses montrent que les chances d'être en emploi ou d'occuper un emploi à temps plein sont plus grandes pour les résidants des collectivités des Premières Nations du Québec que pour ceux des collectivités avoisinantes, une fois les autres caractéristiques, dont l'accès à la scolarité, tenues en compte. La discordance entre nos résultats et ceux des recherches antérieures peut s'expliquer, d'une part, par le fait que nous n'avons conservé que les collectivités à proximité des communautés des Premières Nations et, d'autre part, par le fait que les recherches antérieures ne tiennent pas compte de l'effet d'autres facteurs, dont la scolarité, sur l'accès à l'emploi.

Ainsi, en tant que groupe, les résidants des collectivités des Premières Nations sont désavantagés par rapport à leurs voisins immédiats d'abord et avant tout pour ce qui est de l'accès à une alphabétisation fonctionnelle et à un logement en bon état et comportant suffisamment de pièces. Le régime juridique particulier auquel sont soumises les collectivités des Premières Nations constitue une piste d'explication de cette situation.

L'autochtonité, mesurée ici par l'auto-identification, est souvent mise de l'avant implicitement dans les recherches quantitatives sur les conditions autochtones. L'intérêt de ce facteur se situe dans le fait qu'il nous renseigne sur la présence de dynamiques historiques, de racisme ou de discrimination à l'égard des Amérindiens, difficilement quantifiables. Les résultats de cette recherche montrent que l'autochtonité explique peu de chose une fois les autres caractéristiques prises en compte, sa plus forte contribution étant d'un demi-point de pourcentage pour ce qui est de l'alphabétisation fonctionnelle ou de la possession d'un diplôme d'études secondaires. Les travailleurs amérindiens apparaissent même légèrement plus susceptibles d'occuper un 
emploi à temps plein, une fois les facteurs pertinents contrôlés. Ces résultats mettent en lumière le fait que les différences d'emploi et de revenu entre Amérindiens et nonAmérindiens se situent d'abord dans l'inégalité des chances d'accès à l'éducation, celle-ci constituant une condition d'accès à un emploi bien rémunéré. À chances égales, les Amérindiens s'en sortent aussi bien que les non-Amérindiens vivant dans un environnement similaire. Il faut cependant rappeler que la qualité des emplois n'est pas prise en compte dans les analyses et, qu'à statut d'emploi et à scolarité égaux, l'accès à un revenu élevé est moins fréquent chez les Amérindiens.

\section{LES LIMITES DE L'ÉTUDE}

Une des limites de cette recherche est l'absence d'informations sur la scolarité des parents puisque les recherches montrent qu'habituellement celle-ci a un impact non négligeable sur la scolarité des enfants. Cette information nous renseignerait sur les facteurs historiques liés à l'accès actuel à l'éducation chez les Amérindiens et chez les résidants des communautés isolées en général. Des différences à ce niveau pourraient être un facteur explicatif non négligeable des différences interindividuelles, y compris selon les zones, les regroupements et les types de communauté.

Par ailleurs, nous nous sommes concentrés sur quatre axes. Le choix de ces axes est tributaire de contraintes intrinsèques aux bases de données utilisées. Les enquêtes censitaires ne permettent pas d'explorer d'autres champs pertinents à l'étude des conditions sociales telles que l'importance des liens de solidarité au sein des communautés, la santé, etc. Il existe donc des limites relatives aux thèmes étudiés et aux variables disponibles pour en rendre compte.

D’autres limites ont trait à la population étudiée. Certaines communautés sont absentes des analyses puisqu'elles n'ont pas été dénombrées dans l'enquête censitaire de 2001. C'est le cas des trois collectivités mohawks (Akwesasne, Kahnawake et Kanesatake) et de celle de Barriere Lake (Lac-Rapide). Cette situation est particulièrement préoccupante dans le cas des collectivités mohawks qui ne sont plus dénombrées depuis le recensement de 1981. Ces collectivités possèdent des caractéristiques spécifiques, notamment le fait d'être situées près d'un grand centre (Montréal), qui, comme pour Wendake, les distinguent des autres collectivités plus éloignées des grands centres urbains. De plus, notre échantillon étant restreint à certaines communautés, il est impossible d'extrapoler les résultats à l'ensemble des Amérindiens domiciliés au Québec. Notons également que les résultats sont tributaires de la mesure choisie pour quantifier l'autochtonité.

L'ajout dans les bases de données d'informations contextuelles pertinentes aurait pu éclairer la compréhension de certains phénomènes. Toutefois, cela aurait demandé de poursuivre la recherche au-delà du temps disponible. Il aurait été pertinent entre autres d'intégrer des informations comme la présence d'écoles primaires et secondaires dans les communautés, la distance à une école ou à un cégep, la taille du territoire de chaque communauté, la proportion de membres des communautés des Premières Nations vivant hors communautés, etc. Les recherches futures devraient intégrer ces informations. 
En dernier lieu, il aurait été souhaitable de faire des analyses longitudinales. Cela aurait permis d'observer les dynamiques d'évolution des inégalités ainsi que d'offrir un aperçu de l'impact des efforts entrepris par un grand nombre de collectivités des Premières Nations pour améliorer l'accès à l'éducation et les opportunités d'emploi qu'elles offrent. À ce titre, soulignons l'augmentation de la fréquentation scolaire chez les résidants des collectivités des Premières Nations qui est attribuée au contrôle accru des communautés sur leur système d'éducation (Dore et Kulshreshta, 2003). L'introduction d'un critère temporel aurait également permis de vérifier les résultats présentés par Wotherspoon et Satzewich (1993) selon lesquels les inégalités sociales entre les membres d'une même collectivité des Premières Nations se seraient accrues, certains individus ayant accès à des emplois de "qualité», notamment dans l'administration de la réserve, alors que d'autres n'auraient accès qu'à des emplois temporaires/ saisonniers ou moins bien rémunérés.

\section{CONCLUSION}

La recherche quantitative portant sur les conditions socioéconomiques des Amérindiens est caractérisée par la mise en parallèle des situations socioéconomiques des Amérindiens et des non-Amérindiens dans le contexte canadien général. De plus, les études sont généralement descriptives et les autres facteurs potentiellement pertinents pour comprendre les dynamiques d'inégalités sociales ne sont pas contrôlés. Cela a pour incidence de renvoyer une image homogène des Amérindiens, mais aussi des non-Amérindiens.

L'utilisation de régressions logistiques multivariées a permis d'identifier les déterminants des inégalités scolaires, d'emploi, de revenu et de logement. Les analyses montrent que les inégalités scolaires sont en amont des inégalités d'emploi et de revenu. En ce sens, les nombreuses initiatives entreprises par les Premières Nations du Québec pour accroître la scolarisation de leurs membres s'avèrent prometteuses. Les résultats de recherches confirment également les besoins criants en matière de logement dans les collectivités, une question régulièrement soulevée par les représentants des Premières Nations. Cela pourrait cacher d'autres problématiques sous-jacentes, notamment le manque d'espace permettant un développement résidentiel dans certaines communautés ou encore la lourdeur de certaines dispositions légales qui entravent la capacité des communautés des Premières Nations à transformer substantiellement les conditions générales d'existence de leurs résidants.

Enfin, les résultats invitent à repenser la façon dont est présentée la situation socioéconomique des Amérindiens puisque des facteurs autres que l'ethnicité interviennent dans les dynamiques d'inégalités, transcendant et fragmentant les catégories «Amérindien» et «non-Amérindien». Plus spécifiquement, les résultats montrent l'urgence, d'une part, de penser au pluriel les conditions d'existence des Amérindiens, mais aussi des non-Amérindiens, et, d'autre part, d'intégrer à l'analyse des inégalités d'autres facteurs, notamment l'environnement immédiat des communautés. 


\section{RÉSUMÉ}

Les recherches sur les conditions socioéconomiques des Autochtones sont dominées par la mise en parallèle de leur situation avec celle des Allochtones. L'autochtonité ou la résidence dans une collectivité des Premières Nations y sont implicitement mises de l'avant comme principaux facteurs explicatifs. La recherche présentée vise à circonscrire l'apport réel de ces deux facteurs en rendant manifeste la contribution d'autres facteurs pertinents. En utilisant les données censitaires de 2001, en réduisant la comparaison aux communautés voisines de celles des Premières Nations et en recourant aux analyses de régression, la recherche permet de montrer l'importance de l'environnement immédiat et de l'isolement géographique pour comprendre la situation des Premières Nations. Elle montre également l'importance de l'égalité des chances, en premier lieu l'accès à l'éducation, dans l'explication de l'accès à un emploi et à un revenu supérieur. Enfin, elle souligne l'ampleur du problème de surpeuplement des logements vécu dans certaines communautés.

Mots clés: Premières Nations, conditions socioéconomiques, recensement, Québec

\section{ABSTRACT}

Research on Aboriginal people's socioeconomic conditions has been dominated by comparisons of Aboriginals with non-Aboriginals. In these studies, aboriginality and residence in a First Nation community are implicitly put forward as main explanatory factors. This research attempts to assess the real contribution of these two factors by demonstrating the contribution of other relevant factors. Using 2001 census data, restricting the comparison to communities located nearby First Nations communities, and using regression analyses, the analysis underlines the importance of the immediate environment and of geographic isolation in explaining the living conditions of members of the First Nations. Furthermore, it shows the importance of equality of opportunities, mainly in access to education, in the explanation of access to employment and to a higher income. Finally, the research underlines the magnitude of the problem of overcrowded dwellings in some communities.

Key words: First Nations, socioeconomic conditions, census, Quebec

\section{RESUMEN}

Las investigaciones sobre las condiciones socioeconómicas de los autóctonos están dominadas por la comparación de su situación con la de los alógenos. Como principales factores explicativos de ello, se presenta la autoctonidad o la residencia en una colectividad de las Primeras Naciones. La investigación aquí presentada busca circunscribir el aporte real de estos dos factores, haciendo manifiesta la contribución de otros factores pertinentes. Utilizando los datos censales de 2001, y reduciendo la comparación a las comunidades vecinas de las Primeras Naciones, y recurriendo a los análisis de regresión, la investigación permite mostrar la importancia del entorno inmediato y del aislamiento geográfico para comprender la situación de las primeras naciones. Esta muestra igualmente la importancia de la igualdad de oportunidades, en primer lugar el acceso a la educación, para explicar el acceso a un empleo y a un ingreso superior. Por último, esta subraya la amplitud del problema de superpoblación de las viviendas que tienen lugar en ciertas comunidades.

Palabras clave: Primera Naciones, condiciones socioeconómicas, censo, Quebec 


\section{BIBLIOGRAPHIE}

Affaires Indiennes et du Nord Canada - AINC (1997), Socio-Economic Indicators in Indian Reserves and Comparable Communities, 1971-1991, Département de la statistique, Ottawa, 55 p., nº R32181/1991E, au catalogue.

Cheal, D. (2003), "Trouver une niche: la différenciation en fonction de l'âge dans la population active», in Différenciation sociale: modèles et processus, D. Juteau (dir.), Les Presses de l'Université de Montréal, p. 85 à 119.

Ministère de l'Éducation, du Loisir et du Sport (MELS) (2004), «L'éducation des populations scolaires dans les communautés autochtones du Québec», Bulletin statistique de l'éducation, $\mathrm{n}^{\circ} 30$, (www.mels.gouv.qc.ca/stat/bulletin/Bulletin_30.pdf), consulté le 20 juin 2011.

De Silva, A. (1999), «Wage Discrimination Against Natives», Canadian Public Policy, vol. 25, n 1, p. 65-85.

Dore, M. H. I., et S. Kulshreshtha (2003), «The Labor Market and Rural-Urban Differences Among First Nations: the Case of Saskatchewan», The Journal of Socio-Economics, vol.32, p. 147-159.

Duhaime, G. et A. Godmaire (2002), «Les modèles de développement du nord. Analyse exploratoire au Québec isolé», Recherches sociographiques, vol. 43, n², p. 329-351.

Dupuis, R. (1998), La question indienne au Canada, Boucherville, Éditions du Boréal, 127 p.

Dupuis, R. (1995), «Les politiques canadiennes et québécoises relatives aux Autochtones», Actes du colloque Autochtones et Québécois: la rencontre des nationalismes, P. TrudeL (dir.), Recherches amérindiennes au Québec, Cégep du Vieux-Montréal, 28 et 29 avril 1995, p. 56-69.

Édouard, R. (2008), Le développement inégal et la production des conditions de vie. Le cas des Inuit de l'Arctique canadien, thèse de doctorat, Université Laval, 393 p.

George, P. et P. Khun (1994), «The Size and Structure of Native-White Wage Differentials» Canadian Journal of Economics, $\mathrm{n}^{\circ} 27$, p. 20-42.

Gerber, L. M. (1990), «Multiple Jeopardy: A Socio-Economic Comparison of Men and Women Among the Indian, Metis and Inuit Peoples of Canada ", Canadian Ethnic Studies, vol. 22, no 3, p. 69-85.

Guimond, É. (2003), Définitions floues et explosion démographique chez les populations autochtones du Canada de 1986 à 2001, Des gens d'ici. Les Autochtones en milieu urbain, D. Newhouse et E. Peters (dir.), Projet de recherche sur les politiques, Canada, (www.policyresearch.gc.ca/doclib/RP_PWFC_ Guimond-paper_200712_f.pdf), consulté le 2 octobre 2009.

Hacking, I. (2006), Cours «B»: Les choses, les gens et la raison. Deux types de gens: les cibles mouvantes, Collège de France.

Maxim, P., J. White et D. Beavon (2001), «Dispersion and Polarization of Income about Indigenous and non-Indigenous Canadians», Canadian Review of Sociology and Anthropology, vol. 38, n 4, p. 465-476.

McHardy, M. et E. O’Sullivan (2004), Bien-être des collectivités des Premières Nations du Canada: indice de bien-être des collectivités (IBC), 2001, Direction de la recherche stratégique et de l'analyse, Ottawa, AINC.

O’Sullivan, E. et M. McHardy (2004), L'indice du bien-être des collectivités (IBC): évolution de la différence entre le bien-être des collectivités des Premières Nations et celui des autres collectivités canadiennes, Catalogue: R2 349/2004F, Direction de la recherche stratégique et de l'analyse, AINC.

Simard, J.-J. (2003), La Réduction. L'Autochtone inventé et les Amérindiens d'aujourd'hui, Sillery, Septentrion, $430 \mathrm{p}$.

Soutнсотт, C. (2003), «Différenciation sociale selon les régions dans le Canada de demain: différences tendancielles entre régions urbaines et régions rurales pour la prochaine décennie», in Différenciation sociale: modèles et processus, D. Juteau (dir.), Les Presses de l’Université de Montréal, p. 206 à 251.

Statistique Canada (2010), Dictionnaire de recensement de 2006, Ottawa, Approvisionnements et Services Canada, Recensement du Canada de 2006, no 92-569-X au catalogue.

Statistique Canada (2008), Aboriginal Peoples in Canada in 2006: Inuit, Métis and First Nations, 2006 Census. Aboriginal People, 2006 Census, Ottawa, Ministère de l'Industrie, ${ }^{\circ}$ 97-558-XIE au catalogue, $52 \mathrm{p}$. 
Statistique CANAda (2006), Questions du recensement de 2006 et raisons pour lesquelles les questions vous sont posées, Ottawa, $49 \mathrm{p}$.

Statistique Canada (2003), Dictionnaire $d u$ recensement de 2001, Ottawa, Approvisionnements et Services Canada. Recensement du Canada de 2001, $n^{\circ}$ 92-378-XIF au catalogue.

Statistique Canada (2001), Questionnaire de recensement. Ottawa, Approvisionnements et Services Canada. Recensement du Canada de 2001, 32 p. (www.statcan.gc.ca/imdb-bmdi/instrument/3901_ Q2_V2-fra.pdf,), consulté le 12 novembre 2010.

Statistique Canada (1996), Questionnaire de recensement. Ottawa, Approvisionnements et Services Canada. Recensement du Canada de 1996, 32 p.

Statistique Canada (1992), Dictionnaire de recensement de 1991, Ottawa, Approvisionnements et Services Canada. Recensement du Canada de 1991. n ${ }^{\circ}$ 92-301F au catalogue.

Wilkes, R. (2010), «Indigenous People», in B. Едmonston et E. Fong (dir.), The Changing Canadian Population, Montréal, McGill Queen's University Press, p. 313-329.

Wotherspoon, T. (2003), «Les Autochtones, les politiques sociales et la différenciation sociale au Canada», in Différenciation sociale: modèles et processus, D. JutEAu (dir.), Les Presses de l'Université de Montréal, p. 155 à 203.

Wotherspoon, T. et V. SAtzewich (1993), First Nations. Race, Class, and Gender Relations, Scarborough Nelson Canada, 311 p. 


\section{AN NEXE: LISTE DES REGROUPEMENTS DE COLLECTIVITÉS}

GR Communautés

1 Odanak: Pierreville, Saint-François-du-Lac, Saint-Thomas-de-Pierreville, NotreDame-de-Pierreville, Saint-Elphège, Saint-Pie-de-Guire, Yamaska, Yamaska Est

2 Wôlinak: Trois-Rivières, Bécancour, Nicolet, Saint-Célestin, Grand-Saint-Esprit, Saint-Sylvère

3 Pikogan: Amos, Tréceson, Saint-Félix-de-Dalquier, Saint-Mathieu-d'Harricana, Saint-Marc-de-Figuery, Sainte-Gertrude-Manneville, Landrienne

4 Lac-Simon: Senneterre, Val-Senneville* ${ }^{*}$ Val-d'Or*

5 Timiskaming: Notre-Dame-du-Nord, Nédélec, Guérin, Saint-Eugène-deGuigues, Angliers, Saint-Bruno-de-Guigues

6 Winneway et Kicisakik: Laforce, Kitcisakik, Belleterre*, Moffet ${ }^{\star}$

7 Kebaowek et Hunter's Point: Kipawa, Hunter's Point, Témiscaming, SaintÉdouard-de-Fabre ${ }^{* *}$

8 Kitigan Zibi: Maniwaki, Egan-Sud, Déléage, Bois-Franc, Messine ${ }^{\star}$

9 Manawan: Saint-Michel-des-Saints**, Saint-Zénon ${ }^{\star * *}$

10 Obedjiwan et Wemotaci: Parent ${ }^{* *}$, Rivière-Windigo ${ }^{* * *}$

11 Nemiscau, Mistissini, Oujé-Bougoumou et Waswanipi: Chapais ${ }^{\star}$, Baie-James+, Chibougamau**

12 Chisasibi, Wemindji, Eastmain, et Waskaganish: Matagami ${ }^{\star * *}$

13 Whapmagoostui: Kuujjuarapik

14 Wendake: Loretteville, Saint-Émile, Charlesbourg, Val-Bélair, Charlesbourg, Vanier, L'Ancienne-Lorette, Lac-Saint-Charles, Saint-Gabriel-de-Valcartier, LacBeauport

15 Pakuashipi + : Saint-Augustin +, Bonne-Espérance +, Blanc-Sablon +, CôteNord-du-Golfe-du-Saint-Laurent +

16 La Romaine + et Natashquan: Aguanish *, Natashquan

17 Mingan: Lointe-Point-de-Mingan, Rivière-Saint-Jean, Havre-Saint-Pierre ${ }^{\star}$, BaieJohan-Beetz ${ }^{* *}$

18 Uashat et Maliotenam: Sept-Îles, Gallix, Moisie

19 Betsiamites: Raguenau, Colombier, Chute-aux-Outardes ${ }^{\star}$

20 Essipit: Les Escoumins, Les Bergeronnes, Longue-Rive ${ }^{\star}$, Tadoussac $^{\star}$

21 Mashteuiatsh: Roberval, Saint-Prime, Saint-Félicien, Sainte-Hedwidge

22 Gesgapegiag: Maria, Cascapedia-Saint-Jules, New Richmond, Caplan, CarletonSaint-Omer

23 Listuguj: Pointe-à-la-Croix, Ristitgouche-Partie-Sud-Est, Escuminac, Matapédia

24 Kawawachikamach+, Matimekosh+ et Lac John+: Schefferville +

Légende

* À plus de $25 \mathrm{~km}$ de la collectivité des Premières Nations

${ }^{*}$ À plus de $50 \mathrm{~km}$ de la collectivité des Premières Nations

${ }_{* * *}$ Ã plus de $100 \mathrm{~km}$ de la collectivité des Premières Nations

+ Aucun accès routier direct 